Check for updates

Cite this: RSC Adv., 2018, 8, 14589

\title{
3-Nitro-4-(tetrazol-5-yl) furazan: theoretical calculations, synthesis and performance $\uparrow$
}

\begin{abstract}
Zhiyue Han, (D)* Qi Jiang, Zhiming Du, (D) Yupeng Zhang and Yuezhen Yang
The synthesis mechanism of 3-nitro-4-(tetrazol-5-yl)furazan (NTZF) was calculated by Gaussian 09 for the first time, and NTZF was successfully synthesized based on the theoretical design. Its ionic salts (RbNTZF and CsNTZF) were synthesized and studied by single-crystal X-ray diffraction firstly. The thermal stability of NTZF was investigated by TG-DSC and the kinetic data of thermal decomposition were calculated. The sensitivity of NTZF was measured. The formation heat, detonation velocity $(D)$ and detonation pressure $(P)$ of NTZF were calculated. NTZF is insensitive to impact and friction (impact $>40 \mathrm{~J}$, friction $>$ $360 \mathrm{~J}$ ) and has higher detonation velocity and pressure ( $D=7.838 \mathrm{~km} \mathrm{~s}^{-1}, P=27.32 \mathrm{GPa}$ ) compared to TNT ( $D=6881 \mathrm{~m} \mathrm{~s}^{-1}, P=19.5 \mathrm{GPa}$ ). NTZF has appropriate sensitivity and detonation performance, so it can be used as a low explosive and gas generant.
\end{abstract}

Received 28th March 2018

Accepted 11th April 2018

DOI: $10.1039 / \mathrm{c} 8 \mathrm{ra02682c}$

rsc.li/rsc-advances

\section{Experimental section}

\section{Introduction}

As a new type of energetic materials, nitrogen-rich compounds contain a high enthalpy of formation and high energy density. Their molecular structures include a large number of chemical bonds $\mathrm{N}-\mathrm{N}, \mathrm{C}-\mathrm{N}, \mathrm{N}=\mathrm{N}, \mathrm{C}-\mathrm{N}$. Some energetic nitrogen-rich compounds have been synthesized for application in the field of military chemistry and explosion safety, such as high-energy insensitive explosives, ${ }^{\mathbf{1 , 2}}$ small propulsion system solid fuels, ${ }^{\mathbf{3}, 4}$ smokeless pyrotechnics, ${ }^{5,6}$ gas generators, ${ }^{7,8}$ flame low temperature fire extinguishing agents ${ }^{9,10}$ and so on.

The molecular formula of NTZF is $\mathrm{C}_{3} \mathrm{~N}_{7} \mathrm{HO}_{3}$. The nitrogen content of it is $53.55 \%$. The structure of it is very stable because of the $\pi$-conjugate formed by the heterocyclic system. Thus, the NTZF is a stable energetic compound which has good application prospects in the field of explosives. NTZF was synthesized for the first time by Wang ${ }^{11}$ with ATZF as the raw material. However, the synthesis mechanism of NTZF hasn't been studied to date.

In this study, the synthesis mechanism of NTZF was studied by theoretical calculations for the first time, and its structure was characterized by IR, ${ }^{1} \mathrm{H}$ NMR, ${ }^{13} \mathrm{C}$ NMR and EA. Single crystals of its ionic salts were cultivated firstly. The thermal stability was investigated by TG-DSC and friction sensitivity was measured. Additionally, its formation heat, detonation pressure and detonation velocity were calculated.

State Key Laboratory of Explosion Science and Technology, Beijing Institute of Technology, 5 South Zhongguancun Street, Haidian District, Beijing, 100081, China. E-mail: hanzhiyue@bit.edu.cn; Fax: +86-01-68912765; Tel: +86-01-68912765

$\dagger$ Electronic supplementary information (ESI) available. CCDC 1833051 and 1833052. For ESI and crystallographic data in CIF or other electronic format see DOI: $10.1039 / \mathrm{c} 8 \mathrm{ra02682 \textrm {c }}$

\subsection{Synthesis of 3-nitro-4-(tetrazol-5-yl)furazan (NTZF)}

All chemical used reagents and solvents were analytically pure, and purchased commercially.

In this paper, 3-amino-4-(tetrazol-5-yl)furazan (ATZF) was synthesized according to the method in ref. 12. NTZF was synthesized according to the theoretical optimization results:

Concentrated $\mathrm{H}_{2} \mathrm{SO}_{4}(4 \mathrm{~mL})$ and ATZF $(0.76 \mathrm{~g}, 5 \mathrm{mmol})$ were added to a three-necked flask at room temperature. The reaction mixture was stirred until the solid had dissolved. $\mathrm{Na}_{2} \mathrm{WO}_{4}$ (2.5 g, $7.5 \mathrm{mmol}$ ) was added to $30 \% \mathrm{H}_{2} \mathrm{O}_{2}(24 \mathrm{~mL})$ and the mixture was stirred gently at $-10{ }^{\circ} \mathrm{C}$, until the solid had completely dissolved. The solution was added dropwise to the three-necked flask where a vigorous exothermic reaction occurred. The temperature of the reaction was adjusted to $35{ }^{\circ} \mathrm{C}$ and keep $3 \mathrm{~h}$. The resulting solution was filtered and extracted with acetate $(20 \mathrm{~mL} \times 3)$. The yellow-green product of NTZF were washed with saturated brine, dried and evaporated. Yield $77.50 \% . \mathrm{C}_{3} \mathrm{~N}_{7} \mathrm{HO}_{3}$ : calcd C 20.04, N 52.12, $\mathrm{H} 0.87 \%$; found: $\mathrm{C}$ 19.68, N 53.55, H 0.55\%; IR (KBr, $\nu \mathrm{cm}^{-1}$ ): 1566.77, 1328.01 $\left(-\mathrm{NO}_{2}\right)$, 3095.65, 1144.77, 1114.42 (Tetrazole ring), 1471.58, 1397.51, 996.10 (Furan ring). ${ }^{1} \mathrm{H}$ NMR (DMSO-d ${ }_{6}, \delta / \mathrm{ppm}$ ): 12.17 (s, 1H). ${ }^{13} \mathrm{C}$ NMR (DMSO-d 6 , $\left.\delta / \mathrm{ppm}\right): 160.02,147.37,141.34$.

RbNTZF and CsNTZF: the synthesis of RbNTZF/CsNTZF was carried out from NTZF by reaction with rubidium/cesium. $1.83 \mathrm{~g}$ (10 mmol) NTZF was added in a three-necked flask with $20 \mathrm{~mL}$ of water at $40{ }^{\circ} \mathrm{C}$. After stirred fully to dissolve, the mixture was added $1.15 \mathrm{~g}$ (5 mmol) rubidium carbonate $/ 1.63 \mathrm{~g}(5 \mathrm{mmol})$ cesium carbonate. The reaction produced a large number of bubbles. After fully responsed to clarification, the reaction liquid was filtered and extracted. The filtrate was mixed with 
toluene by certain proportion. The product was steamed at $60{ }^{\circ} \mathrm{C}$ and dried backspin steamed.

\subsection{Single crystal cultivation}

A portion of the solution extracted during the above reaction was removed. The sample was placed in a clean beaker after filtering. The beaker was placed in a thermostatic incubator for 3 days until the colorless crystal which can be used for single crystal test appear.

\subsection{Measurements}

Infrared spectra (IR) was recorded with a Nicolet FT-IR6700. Elemental analysis was measured with a Vario EL elemental analyzer. The NMR spectrum was carried out with a DRX500 Bruker NMR spectrometer. Single crystal structure characterization was determined by Rigaku 742+ single crystals diffractometer.

Differential scanning calorimeter (DSC) and thermogravimetric analysis (TG) were carried out with a STARe system and a CDR-4P differential scanning calorimeter and thermogravimetric analyzer. TG-DSC of ATZF was measured under nitrogen

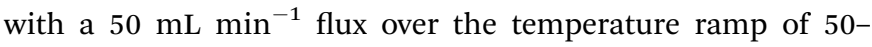
$400{ }^{\circ} \mathrm{C}$, at a heating rate of $2,5,10,20{ }^{\circ} \mathrm{C} \min ^{-1}$.

The impact and the friction sensitivities were tested according to BAM standard methods. The flame sensitivity was determined by HGY-1 flame sensitivity instrument. The sample mass used for each test was $20 \pm 2 \mathrm{mg}$. The sample was compressed into a fire cap at $58.8 \mathrm{MPa}$ and the cap was ignited by the standard black powder column. The $50 \%$ ignition height indicated the friction sensitivity.

\section{Result and discussion}

\subsection{Theoretical calculation}

3.1.1 Structure of NTZF. The structure of NTZF was optimized with B3LYP/6-311 ++ G ** (ref. 13) using the Gaussian 09 software package. ${ }^{14}$ The geometric structure of the NTZF molecule is shown in Fig. 1 and the bond lengths and bond angles are shown in ESI Tables 1 and $2 . \dagger$

Based on the principle of minimum energy, the lone pair electrons on one oxygen atom of the nitro group repulsively

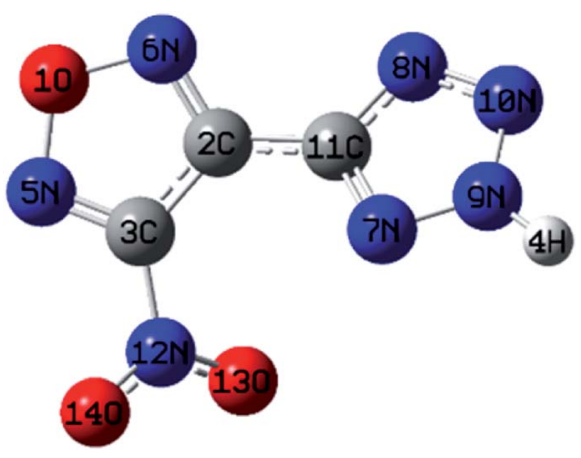

Fig. 1 Geometry of NTZF.
Table 1 NBO charge of NTZF

\begin{tabular}{llll}
\hline Atom & Charge/a.u. & Atom & Charge/a.u. \\
\hline O1 & -0.11 & $\mathrm{~N} 8$ & -0.23 \\
C2 & 0.07 & $\mathrm{~N} 9$ & -0.13 \\
C3 & 0.23 & $\mathrm{~N} 10$ & -0.03 \\
H4 & 0.42 & $\mathrm{C} 11$ & 0.25 \\
N5 & -0.05 & $\mathrm{~N} 12$ & 0.46 \\
N6 & -0.03 & $\mathrm{O} 13$ & -0.31 \\
N7 & -0.23 & $\mathrm{O} 14$ & -0.30
\end{tabular}

opposes the lone pair of nitrogen atoms in tetrazole. The furazan ring, tetrazole ring and nitro in NTZF are not coplanar. The bond length of furazan ring is between 1.30-1.43 $\AA$ and the bond angles are similar to each other. Therefore, it tends to form a stable five-membered ring structure. The repulsion between the lone pair of the oxygen atom and nitrogen atoms makes the bond angles of the N5-O1-N4 in the furazan ring become larger. What's more, the bond length in the tetrazole ring is between 1.30-1.36 $\AA$ and the bond angles are similar. It is also a stable five-membered ring structure. In summary, the optimized molecular structure of NTZF is stable.

3.1.2 NBO. Natural bond orbital (NBO) analysis was performed on the geometry of the optimized NTZF at B3LYP/6-311 $++\mathrm{G} * *$ level. The NBO charge distribution of each atom in NTZF was obtained (Table 1). The heavy electron attracting effect of nitro in NTZF makes the $\mathrm{C} 3$ atom positively charged. $\mathrm{N} 12$ atom is also positively charged due to the electronegativity in oxygen is greater than that in nitrogen. The electronegativity of the oxygen in the furazan ring is larger and affected by electrons in the 4 and 5 positions. Therefore, the negative charge in N5 and N6 of the ring is less. The electronwithdrawing ability of nitro in $\Pi_{4}^{3}$ system is larger than that in $\pi$-conjugated tetrazole, therefore, the positive charge carried by C3 is much larger than C2. The conjugate system of the tetrazole ring accepts the electron the hydrogen atom was supplied with, so that the hydrogen with the positive charge is more easily ionized.

3.1.3 ESP. Fig. 2 shows the ESPs for the 0.001 electronic per bohr ${ }^{3}$ isosurfaces of electron density elaluated at the B3LYP/6$311++\mathrm{G}^{* *}$ level. The colors range from $-3.00 \times 10^{-2}$ to $+5.00 \times$

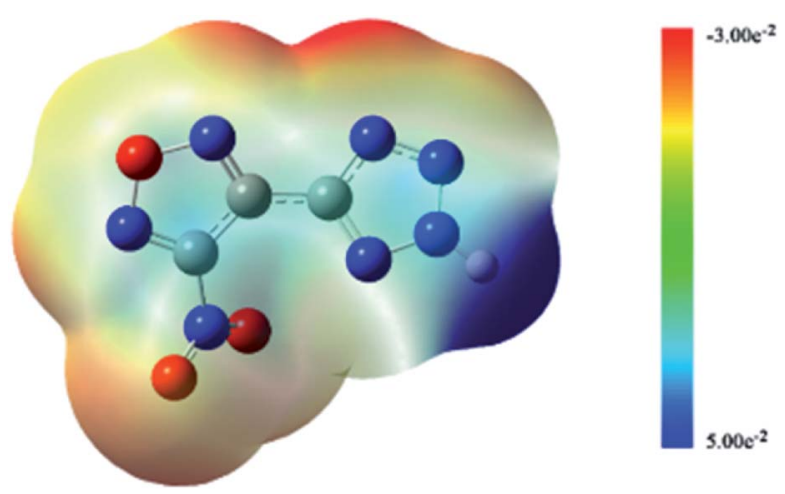

Fig. 2 ESPs of NTZF 
$10^{-2} \mathrm{eV}$ with dark blue denoting extremely electron-deficient regions $\left[V(r) \geq 5.00 \times 10^{-2}\right]$ and red denoting electron-rich regions $\left[V(r) \leq-3.00 \times 10^{-2}\right]$.

As shown in Fig. 2, the hydrogen atom on the tetrazole ring can easily lose, because the positive potential of it is the maximum. It shows that the NTZF can become an anion. The nitro and oxygen atom of furazan, nitrogen atom of tetrazole and nitro group are negative potential distribution. Among them, the negative potential of the nitroxide, the nitrogen atom of the furazan ring and part of the nitrogen atoms on the tetrazole ring are close to the maximum. Therefore, these atoms all have coordination ability. The results of ESP of NTZF are consistent with the results of NBO.

3.1.4 HOMO-LOMO. Fig. 3 shows the HOMO-LUMO orbitals of the NTZF based on the molecular frontier orbital theory. When the electrons in NTZF are stimulated by external energy, the electrons in the tetrazolyl will transfer toward the furazan ring and the nitro group. The energy of the HOMO orbitals of the NTZF is $-9.07 \mathrm{eV}$ and the energy of the LUMO orbitals is $-3.66 \mathrm{eV}$. The difference between the two molecular orbitals $(\Delta E=5.42 \mathrm{eV})$ is the energy required for the electrons in the molecule to excite from the ground state to the excited state. Above all, the molecule is relatively stable.

\subsection{Synthesis mechanism of NTZF}

The oxidizing of $\mathrm{H}_{2} \mathrm{O}_{2}$ is reflected in its own heterogeneous ability. As is shown in Fig. 4, the hydrogen atoms in $\mathrm{H}_{2} \mathrm{O}_{2}$ move to the oxygen atom and undergo isomerization, forming a water

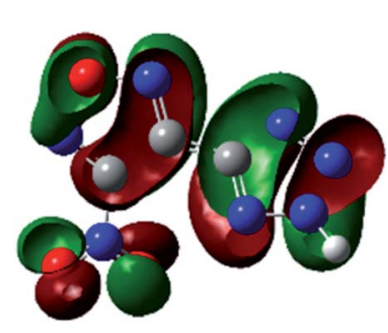

HOMO

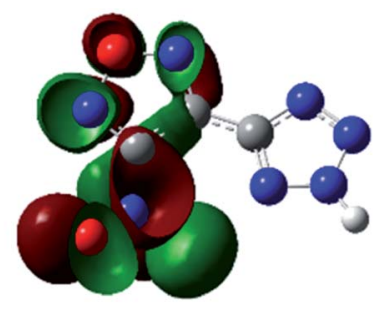

LUMO
Fig. 3 The HOMO, LUMO orbitals of NTZF.

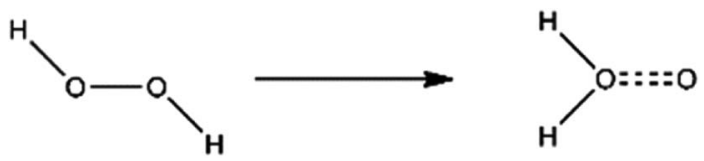

Fig. 4 Isomerization of $\mathrm{H}_{2} \mathrm{O}_{2}$.

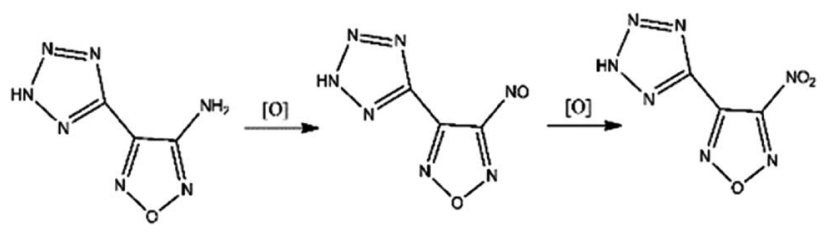

Fig. 5 Nitrification process of ATZF.

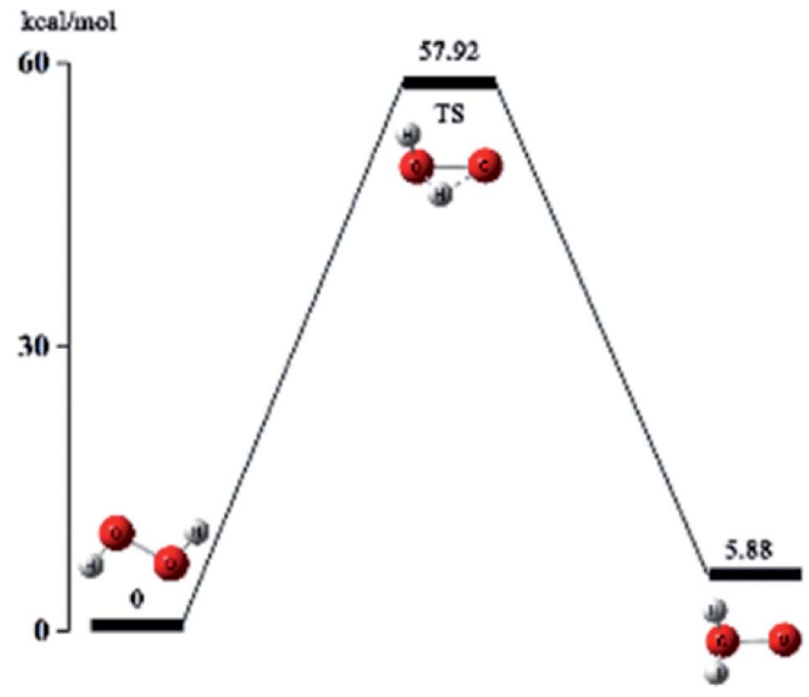

Fig. 6 Isomerization of $\mathrm{H}_{2} \mathrm{O}_{2}$.

molecule with one active oxygen atom. The active oxygen atom is the key to oxidation. The amino-group $\left(-\mathrm{NH}_{2}\right)$ in ATZF is oxidized by $\mathrm{H}_{2} \mathrm{O}_{2}$ to form nitroso-group $(-\mathrm{N}=\mathrm{O})$, which is further oxidized by $\mathrm{H}_{2} \mathrm{O}_{2}$ to form a nitro group (Fig. 5).

Based on the above conjecture, the Gaussian 09 package was used to explore the transition state of the nitration reaction via the TST. The potential energy curves of the $\mathrm{H}_{2} \mathrm{O}_{2}$ isomerization and the nitration reaction are shown in Fig. 6 and 7 .

As is shown in Fig. 6, hydrogen atom at one end of $\mathrm{H}_{2} \mathrm{O}_{2}$ molecule break away from the oxygen atom which was bonded to and migrate to another oxygen atom and then reach to the transition state TS. Eventually, the hydrogen atom in $\mathrm{H}_{2} \mathrm{O}_{2}$ completely bonds with the oxygen atom and forms a stable structure of water molecules with an active oxygen atom. The energy of the isomeric $\mathrm{H}_{2} \mathrm{O}_{2}$ molecule is $5.88 \mathrm{kcal} \mathrm{mol}^{-1}$ higher than the original $\mathrm{H}_{2} \mathrm{O}_{2}$ molecule. It indicates that the reaction is an endothermic reaction. Therefore, the reaction requires an appropriate temperature and catalyst to activate the $\mathrm{H}_{2} \mathrm{O}_{2}$ molecules.

As Fig. 7(a) shows, the amino group in the ATZF molecule undergoes a nucleophilic attack by the active oxygen, and the reaction reaches the transition state TS1. TS1 is the process of active oxygen dissociating from the binding of water molecules in $\mathrm{H}_{2} \mathrm{O}_{2}$ to the nitrogen atom in the amino group. After the nucleophilic addition of the oxygen atom, the amino group formed a tetrahedral configuration, and the system reached a stable point $\mathrm{S} 1$. This is the first oxidation of the $\mathrm{H}_{2} \mathrm{O}_{2}$. Since the new configuration in the stable point S1 structure is not stable enough and the oxygen atom carry a large amount of negative charges, a hydrogen atom on the amino group migrates to the oxygen atom and easily formed a hydroxyl structure. The reaction reached the second transition state TS2. Then, the hydrogen atom detaches from nitrogen atom to bond with the oxygen atom. The system reach the second stable point $\mathrm{S} 2$. The energy of the S2 is $27.59 \mathrm{kcal} \mathrm{mol}^{-1}$ lower than the ATZF, and the reaction is a heat release process. 


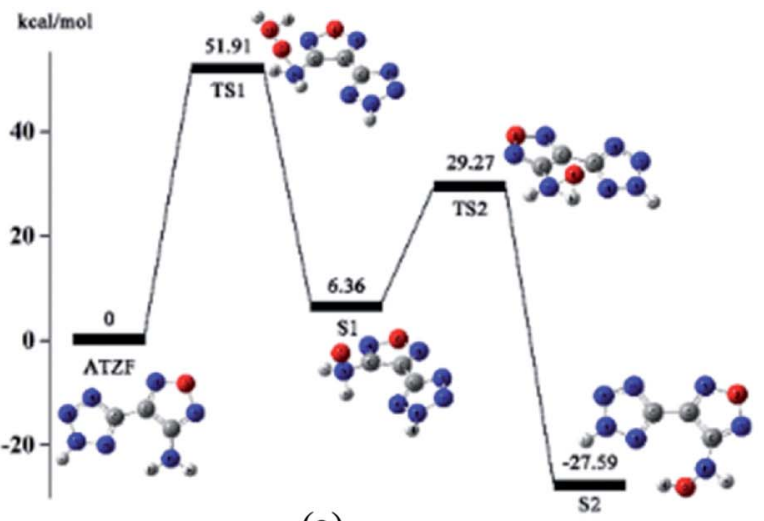

(a)

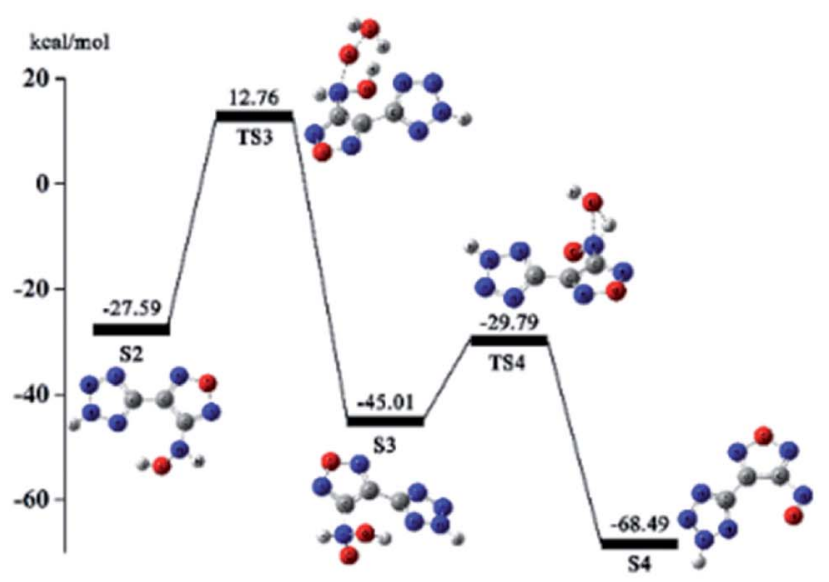

(b)

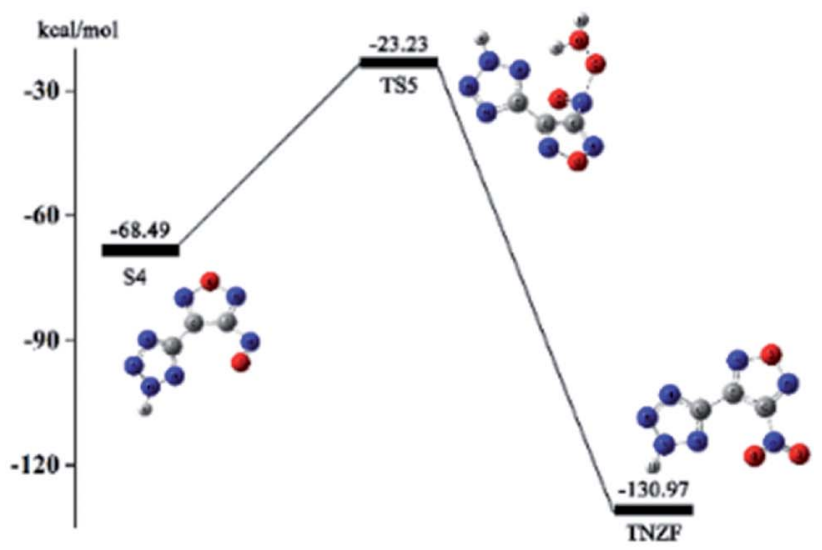

(c)

Fig. 7 Synthesis of NTZF.

Fig. 7(b) shows the hydroxyamino in the molecule of S2 undergoes the second nucleophilic attack by the active oxygen, and reaction reaches the transition state TS3. TS3 is the process of active oxygen dissociating from the binding of water molecules in isomeric $\mathrm{H}_{2} \mathrm{O}_{2}$ to the nitrogen atom in the hydroxyamino. Then, the active oxygen in $\mathrm{H}_{2} \mathrm{O}_{2}$ completely breaks free from the another oxygen atom and forms a stable chemical bond with the nitrogen atom on the hydroxyl amine group. This is the second oxidation of $\mathrm{H}_{2} \mathrm{O}_{2}$. After the nucleophilic addition of the oxygen atom and nitrogen atom, the hydroxyamino formed a new tetrahedral configuration, and the system reached the third stable point S3. Since the hydrogen atom on the imino group is attracted by the charge on the oxygen atom of the hydroxyl and the newly added reactive oxygen species, a hydrogen atom on the imino group can easily migrate to the oxygen atom on the hydroxyl group and forms a water molecule. The reaction reached the transition state TS4. After that, the hydrogen atom on the oxidized imino group is transferred to the oxygen atom of the hydroxyl group to form a water molecule which is detached from the entire molecular system. The system reaches the fourth stable point S4. The energy of the S4 is $68.49 \mathrm{kcal} \mathrm{mol}^{-1}$ lower than the ATZF, that is, nitrite reaction will release a lot of heat.

Fig. 7(c) shows the nitroso-group in the molecule of S4 undergoes the third nucleophilic attack by the active oxygen of the isomeric $\mathrm{H}_{2} \mathrm{O}_{2}$, and reaction reaches the transition state TS5. TS5 is the process of active oxygen atom dissociating from the binding of water molecules in isomeric $\mathrm{H}_{2} \mathrm{O}_{2}$ to the nitrogen atom in the nitroso-group. Then, the active oxygen atom detaches from isomeric $\mathrm{H}_{2} \mathrm{O}_{2}$ completely to bond with the nitrogen atom of the nitroso-group to form a more stable $\Pi_{4}^{3}$ system. At this point, the nitration of the amino group is completed and the system reaches the stable point S6 to generate NTZF. The energy of NTZF is $130.97 \mathrm{kcal} \mathrm{mol}^{-1}$ lower than the energy of the ATZF, that is, the reaction is a dramatic exothermic reaction.

Through the discussion of the above nitrification mechanism, it was found that the three attacks of active oxygen atom in isomeric $\mathrm{H}_{2} \mathrm{O}_{2}$ is correspond to the three transition states TS1, TS3, and TS5. The three transition states are the key steps affect the reaction rate in the entire nitrification process.

\subsection{Crystal structure determination}

3.3.1 RbNTZF and CsNTZF. A colorless flaky crystal with dimensions of $0.2 \times 0.05 \times 0.05 \mathrm{~mm}$ was chosen for X-ray determination. The related parameter of the crystal are shown in Tables 2 and 3. Fig. 8 and 9 show the structure of ionicsalts. Fig. 10 and 11 show the crystal accumulation diagram.

\subsection{Stability of NTZF}

3.4.1 Thermal analysis. The TG-DSC curves of NTZF with a heating rate of $10{ }^{\circ} \mathrm{C} \mathrm{min}{ }^{-1}$ is shown in Fig. 12. In the DSC curve, an endothermic peak appears at $122.83{ }^{\circ} \mathrm{C}$, while the TG curve remains unchanged, the reason is that NTZF undergoes a phase change and endothermically melts. The curve's melting range is short and the peak is sharp, it indicates that the product is pure. In the TG curve, there is a sharply mass loss platform, the mass loss rate is $81.36 \%$, corresponding to the exothermic decomposition peak temperature of the DSC curve at $176.83-248.50{ }^{\circ} \mathrm{C}$. The residual solid is assumed to be the carbon element in the molecule (19.68\% of theoretical value). 
Table 2 Crystal parameter of RbNTZF

\begin{tabular}{|c|c|}
\hline Identification code & RbNTZF \\
\hline Empirical formula & $\mathrm{C}_{3} \mathrm{~N}_{7} \mathrm{O}_{3} \mathrm{Rb}$ \\
\hline Formula weight & 267.57 \\
\hline Temperature/K & 153.15 \\
\hline Crystal system & Orthorhombic \\
\hline Space group & $\mathrm{Pca2}_{1}$ \\
\hline$a / \AA$ & $10.203(2)$ \\
\hline$b / \AA$ & $9.936(2)$ \\
\hline$c / \AA$ & $7.3949(15)$ \\
\hline$\alpha /^{\circ}$ & 90 \\
\hline$\beta /^{\circ}$ & 90 \\
\hline$\gamma /{ }^{\circ}$ & 90 \\
\hline Volume $/ \AA^{3}$ & $749.7(3)$ \\
\hline$Z$ & 4 \\
\hline$\rho_{\text {calc }} / \mathrm{g} \mathrm{cm}^{-3}$ & 2.37 \\
\hline$\mu / \mathrm{mm}^{-1}$ & 6.599 \\
\hline$F(000)$ & 512.0 \\
\hline Crystal size $/ \mathrm{mm}^{3}$ & $0.2 \times 0.05 \times 0.05$ \\
\hline Radiation & $\operatorname{MoK} \alpha(\lambda=0.71073)$ \\
\hline $2 \theta$ range for data collection $/^{\circ}$ & 4.1 to 54.958 \\
\hline Index ranges & $\begin{array}{l}-13 \leq h \leq 7, \\
-11 \leq k \leq 12,-9 \leq l \leq 9\end{array}$ \\
\hline Reflections collected & 2854 \\
\hline Independent reflections & $1601\left[R_{\mathrm{int}}=0.0283, R_{\mathrm{sigma}}=0.0387\right]$ \\
\hline Data/restraints/parameters & $1601 / 1 / 127$ \\
\hline Goodness-of-fit on $F^{2}$ & 1.253 \\
\hline Final $R$ indexes $[I \geq 2 \sigma(I)]$ & $R_{1}=0.0315, \mathrm{w} R_{2}=0.0923$ \\
\hline Final $R$ indexes [all data] & $R_{1}=0.0350, \mathrm{w} R_{2}=0.1127$ \\
\hline
\end{tabular}

Table 3 Crystal parameter of CsNTZF

\begin{tabular}{|c|c|}
\hline Identification code & CsNTZF \\
\hline Empirical formula & $\mathrm{C}_{3} \mathrm{~N}_{7} \mathrm{O}_{3} \mathrm{Cs}$ \\
\hline Formula weight & 315.01 \\
\hline Temperature/K & 153.15 \\
\hline Crystal system & Orthorhombic \\
\hline Space group & $\operatorname{Pca2}_{1}$ \\
\hline$a / \AA$ & $10.376(2)$ \\
\hline$b / \AA$ & $10.128(2)$ \\
\hline$c / \AA$ & $7.6675(15)$ \\
\hline$\alpha /^{\circ}$ & 90 \\
\hline$\beta /^{\circ}$ & 90 \\
\hline$\gamma /{ }^{\circ}$ & 90 \\
\hline Volume $/ \AA^{3}$ & $805.8(3)$ \\
\hline$Z$ & 4 \\
\hline$\rho_{\text {calc }} / \mathrm{g} \mathrm{cm}^{-3}$ & 2.60 \\
\hline$\mu / \mathrm{mm}^{-1}$ & 4.592 \\
\hline$F(000)$ & 584.0 \\
\hline Crystal size $/ \mathrm{mm}^{3}$ & $0.12 \times 0.08 \times 0.04$ \\
\hline Radiation & $\operatorname{MoK} \alpha(\lambda=0.71073)$ \\
\hline $2 \theta$ range for data collection ${ }^{\circ}$ & 4.022 to 54.946 \\
\hline Index ranges & $\begin{array}{l}-13 \leq h \leq 13 \\
-13 \leq k \leq 7,-6 \leq l \leq 9\end{array}$ \\
\hline Reflections collected & 3059 \\
\hline Independent reflections & $1502\left[R_{\text {int }}=0.0318, R_{\text {sigma }}=0.0366\right]$ \\
\hline Data/restraints/parameters & $1502 / 7 / 127$ \\
\hline Goodness-of-fit on $F^{2}$ & 1.273 \\
\hline Final $R$ indexes $[I \geq 2 \sigma(I)]$ & $R_{1}=0.0301, \mathrm{w} R_{2}=0.0921$ \\
\hline Final $R$ indexes [all data] & $R_{1}=0.0388, \mathrm{w} R_{2}=0.1434$ \\
\hline
\end{tabular}

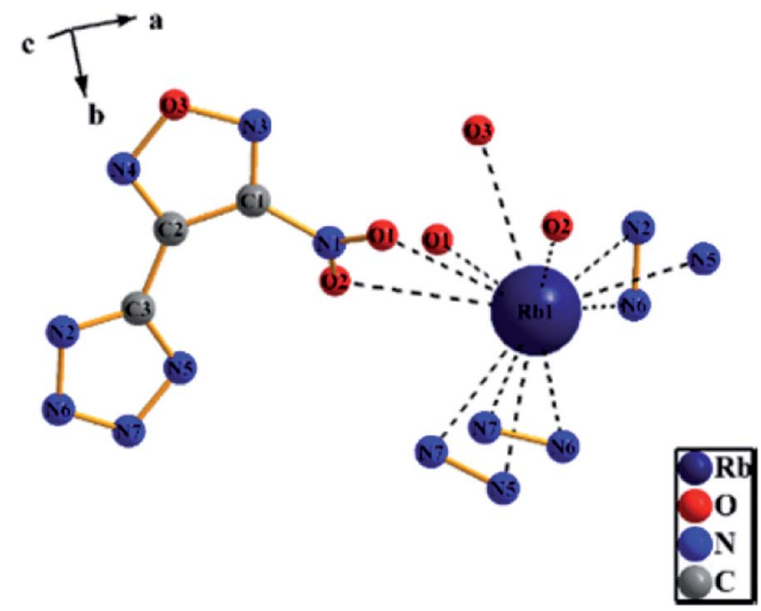

Fig. 8 The structure of RbNTZF.

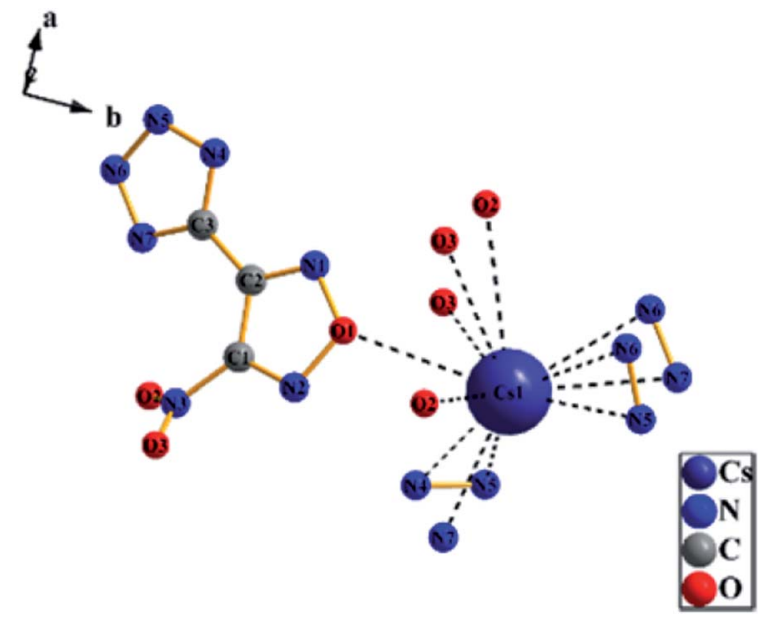

Fig. 9 The structure of CsNTZF.

Subsequently, the carbon atoms oxidize with excess oxygen atoms again, so the TG curve is still slowly declining. For NTZF, the energy release has been completed at $248.50{ }^{\circ} \mathrm{C}$. It indicates that NTZF is an ideal energetic compound with a high concentration of energy release.

DSC curves of NTZF with heating rate 5, 10, 15, and $20{ }^{\circ} \mathrm{C} \min ^{-1}$ are shown in Fig. 13. The kinetic data of NTZF thermal decomposition were calculated by Kissinger method (eqn (1)) and Ozawa method (eqn (2)) respectively. The results are shown in Table 4.

Kissinger equation ${ }^{\mathbf{1 5}}$ :

$$
\ln \left(\frac{\beta}{T_{\mathrm{p}}^{2}}\right)=\ln \left(\frac{A R}{E}\right)-\frac{E}{R}\left(\frac{1}{T_{\mathrm{p}}}\right)
$$

Ozawa equation ${ }^{\mathbf{1 6}}$ :

$$
\lg \beta=\lg \left(\frac{A E}{R G(\alpha)}\right)-2.315-0.4567 \frac{E}{R T_{\mathrm{p}}}
$$



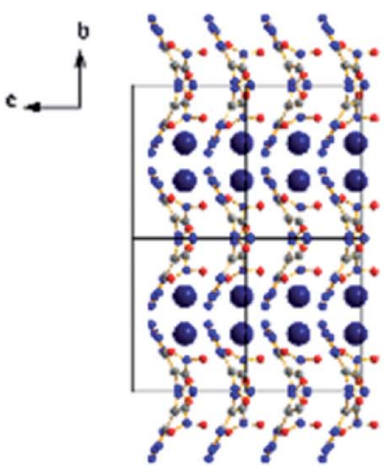
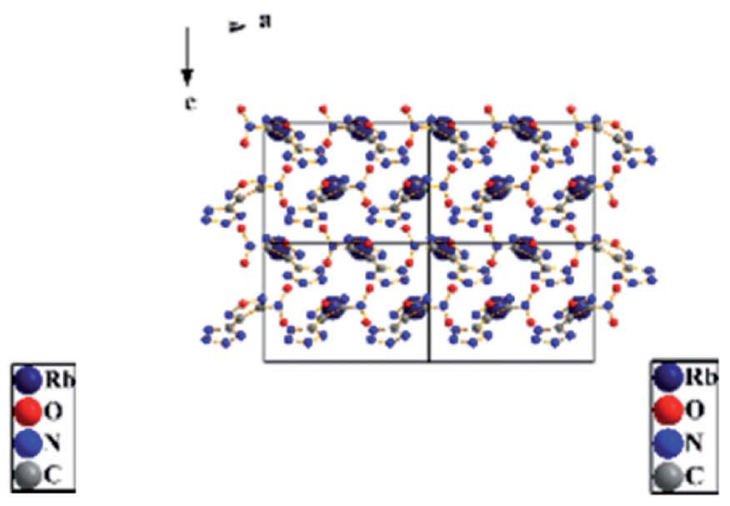

Fig. 10 Crystal accumulation diagram of RbNTZF.
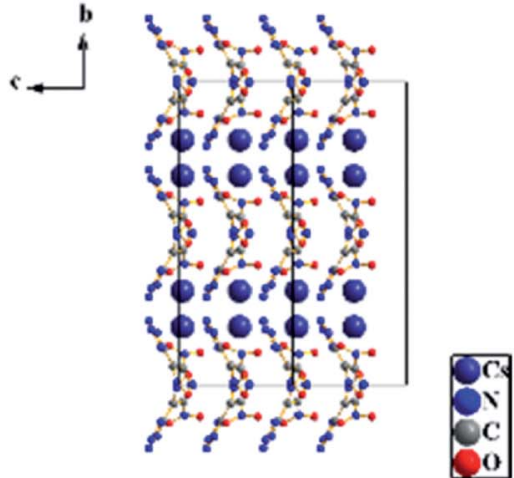

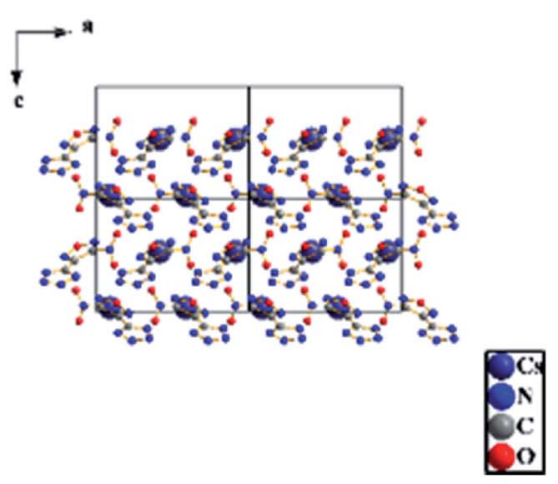

Fig. 11 Crystal accumulation diagram of CsNTZF.

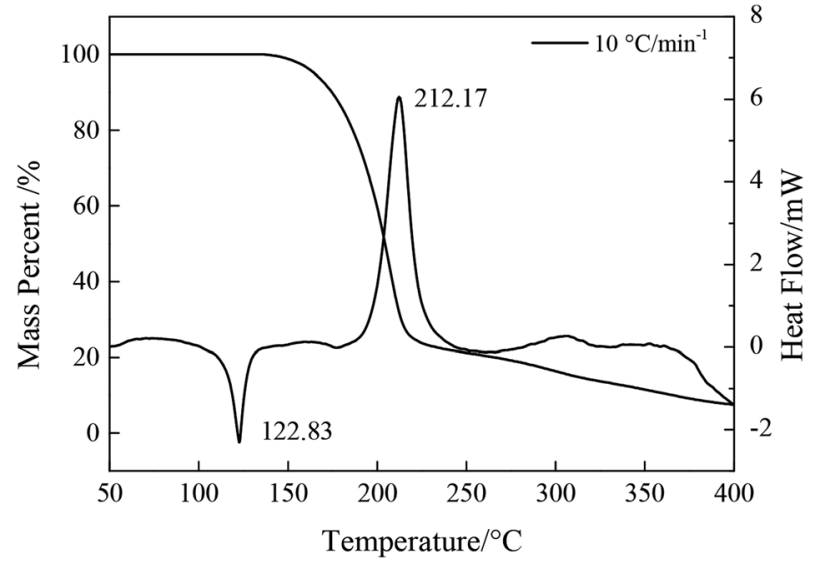

Fig. 12 TG-DSC curves of NTZF with the heating rate $10{ }^{\circ} \mathrm{C} \mathrm{min}^{-1}$.

where $\beta$ is the heating rate $\left[\mathrm{K} \mathrm{min}^{-1}\right], T_{\mathrm{p}}$ is the peak temperature $[\mathrm{K}], E$ is the activation energy $\left[\mathrm{KJ} \mathrm{mol}^{-1}\right], A$ is the pre-exponential factor $\left[\mathrm{s}^{-1}\right]$, and $R$ is the gas constant $\left[\mathrm{J} \mathrm{min}^{-1} \mathrm{~K}^{-1}\right]$.

3.4.2 Sensitivity tests. Table 5 shows the sensitivity data of NTZF. It indicates that NTZF is insensitive toward impact and friction, which are all higher than TNT (impact, $15 \mathrm{~J}$; friction, $353 \mathrm{~N}$ ) and has a certain ignition performance.
3.4.3 Detonation pressure and detonation velocity. The relevant electron energy parameters of NTZF are obtained by analyzing the optimized NTZF geometry results at the B3LYP/6$311++\mathrm{G}^{* *}$ level (Table 6). By substituting the parameters into eqn (3) and (4), ${ }^{17}$ the standard molar formation enthalpy of NTZF at $298 \mathrm{~K}$ can be calculated. Then, the standard molar enthalpy of formation is introduced into eqn (5)-(7). ${ }^{\mathbf{1 8 , 1 9}}$ The

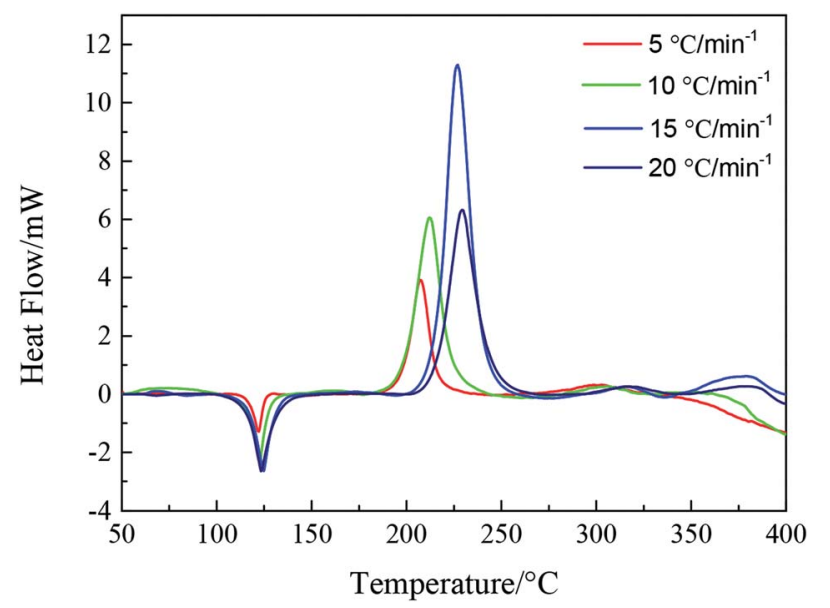

Fig. 13 DSC curves of NTZF with the heat rate 5, 10, 15, and $20^{\circ} \mathrm{C} \min ^{-1}$ 
Table 4 Thermal decomposition kinetics data of NTZF

\begin{tabular}{|c|c|c|c|c|c|c|}
\hline & Peak temperature $T_{\mathrm{p}} /{ }^{\circ} \mathrm{C}$ & \multicolumn{3}{|l|}{ Kissinger } & \multicolumn{2}{|l|}{ Ozawa } \\
\hline 10 & 212.83 & & & & & \\
\hline 15 & 227.00 & & & & & \\
\hline 20 & 229.33 & & & & & \\
\hline
\end{tabular}

Table 5 Sensitivity data of NTZF

\begin{tabular}{lll}
\hline Impact/J & Friction/N & Flame $/ \mathrm{cm}$ \\
\hline$>40$ & $>360$ & 22.50
\end{tabular}

Table 6 Relevant electron energy parameters of NTZF

\begin{tabular}{lll}
\hline$\varepsilon_{0}+\varepsilon_{\text {ZPE }}[$ a.u.] & $\varepsilon_{\text {ZPE }}[$ a.u.] & $H_{\text {corr }}[$ a.u. $]$ \\
\hline-723.71 & 0.07 & 0.09 \\
\hline
\end{tabular}

Table 7 Detonation data of NTZF

\begin{tabular}{lll}
\hline$\Delta_{\mathrm{f}} H^{\circ}(298 \mathrm{~K})\left[\mathrm{kJ} \mathrm{mol}^{-1}\right]$ & $D\left[\mathrm{~m} \mathrm{~s}^{-1}\right]$ & $P[\mathrm{GPa}]$ \\
\hline 256.32 & 7838.17 & 27.32
\end{tabular}

theoretical detonation velocity and detonation pressure of NTZF can be predicted by the semi-empirical $\mathrm{K}-\mathrm{J}$ equation. The formation enthalpy, detonation velocity and detonation pressure are listed in Table 7 . The calculated detonation velocity and detonation pressure are both higher than TNT $\left(D=6881 \mathrm{~m} \mathrm{~s}^{-1}\right.$, $P=19.5 \mathrm{GPa})$.

$$
\begin{aligned}
\Delta_{\mathrm{f}} H^{\circ}(M, 0 \mathrm{~K}) & =\sum_{\text {atoms }} x \Delta_{\mathrm{f}} H^{\circ}(X, 0 \mathrm{~K})-\sum D_{0}(M) \\
& =\sum_{\text {atoms }} x \Delta_{\mathrm{f}} H^{\circ}(X, 0 \mathrm{~K})-\left(\sum_{\text {atoms }} x \varepsilon_{0}(X)-\varepsilon_{0}(M)\right)
\end{aligned}
$$

$$
\begin{aligned}
\Delta_{\mathrm{f}} H^{\circ}(M, 298 \mathrm{~K})= & \Delta_{\mathrm{f}} H^{\circ}(M, 0 \mathrm{~K})+\left(H_{M}^{\circ}(298 \mathrm{~K})-H_{M}^{\circ}(0 \mathrm{~K})\right) \\
& -\sum_{\text {atoms }} x\left(H_{X}^{\circ}(298 \mathrm{~K})-H_{X}^{\circ}(0 \mathrm{~K})\right)
\end{aligned}
$$

$$
\begin{gathered}
P=K \rho^{2} \varphi \\
D=A \varphi^{1 / 2}(1+B \rho) \\
\varphi=N \sqrt{M\left(-\Delta_{E} H\right)}
\end{gathered}
$$

\section{Conclusions}

(1) Based on the density functional theory (DFT), the molecular structure of NTZF is designed and optimized. The synthesis mechanism of NTZF is explored by the transition state theory for the first time.

(2) NTZF was successfully synthesized based on the theoretical design. Its ionicsalts (RbNTZF and CsNTZF) were synthesized and studied by single-crystal X-ray diffraction firstly.

(3) The stability and detonation performance of NTZF were studied. It has good thermal stability (the exothermic decomposition peak temperature is at $176.83-248.50{ }^{\circ} \mathrm{C}$ ). NTZF is insensitive toward impact and friction (impact $>40 \mathrm{~J}$, friction $>$ $360 \mathrm{~J})$. The formation heat, detonation velocity and detonation pressure is $256.32 \mathrm{~kJ} \mathrm{~mol}^{-1}, 7.838 \mathrm{~km} \mathrm{~s}^{-1}$ and $27.32 \mathrm{GPa}$.

These indicate that NTZF is an energetic heterocyclic compound with low sensitivity which has broad application prospects in low explosives and gas generant.

\section{Conflicts of interest}

There are no conflicts to declare.

\section{Acknowledgements}

This work has been supported by the Fundamental Research Funds for the Central Universities.

\section{References}

1 D. Kumar, G. H. Imler, D. A. Parrish and J. M. Shreeve, A Highly Stable and Insensitive Fused Triazolo-Triazine Explosive (TTX), Chem.-Eur. J., 2017, 23, 1743-1747.

2 M. Hiskey, D. Chavez and D. L. Naud, Insensitive HighNitrogen Compounds, Report LA-UR-01-1493, Los Alamos National Laboratory, Los Alamos, NM, USA, 2001.

3 T. Chujo, O. Mori and Y. Kubo, Development of solid-gas equilibrium propulsion system for small spacecraft, Acta Astronaut., 2017, 140, 133-139.

4 A. N. Ali, S. F. Son, M. Hiskey and D. L. Naud, Novel HighNitrogen Propellant Use in Solid Fuel Micropropulsion, $J$. Propul. Power, 2004, 20, 120.

5 D. Chavez, M. Hiskey and D. L. Naud, High-Nitrogen Fuels for Low Smoke Pyrotechnics, J. Pyrotech., 1999, 10, 17-36. 
6 M. Hiskey, D. Chavez and D. L. Naud, Low Smoke Pyrotechnic Compositions, US Pat. 6312537 B1, The Regents of the University of California, Davis, CA, USA, 2001.

7 P. S. Khandhadia, S. P. Burns and G. K. Williams, High Gas Yield Nonazide Gas Generants, US Pat. 6210505 B1, Automotive Systems Laboratory, Inc., Farmington Hills, MI, USA, 2001.

8 Z. M. Du, Y. H. Zhang, Z. Y. Han and Q. Yao, 4,5-Bis(5tetrazolyl)-1,2,3-triazole: synthesis and performance, Propellants, Explos., Pyrotech., 2015, 40, 954-959.

9 P. S. Khandhadia and S. P. Burns, Thermally Stable Nonazide Automotive Airbag Propellants, US Pat. 6306232, Automotive Systems Laboratory, Inc., Farmington Hills, MI, USA, 2001.

10 G. Holl, T. M. Klapçtke and J. J. Weigand, Preparation of Dihydrazinium Compounds of 5,5'-Azotetrazolate Type, Used in Propellant, Pyrotechnic Formulation, Rocket Fuel or Gas-Generating Composition, Involves Reacting Alkali Metal Azotetrazole and Hydrazinium Salt in Aqueous Medium, DE102005011563-A1, Germany Federal Ministry of Defense, Berlin, Germany, 2005.

11 B. Wang, G. Zhang, H. Huo, Y. Fan and X. Fan, Synthesis, Characterization and Thermal Properties of Energetic Compounds Derived from 3-Amino-4-(tetrazol-5-yl)furazan, Chin. J. Chem., 2011, 29, 919-924.
12 R. Wang, Y. Guo, Z. Zeng, B. Twamley and J. Shreeve, Furazan-functionalized Tetrazolate-based Salts: A New Family of Insensitive Energetic Materials, Chem.-Eur. J., 2009, 15, 2625.

13 J. E. D. Bene, W. B. Person and K. Szczepaniak, Properties of Hydrogen-Bonded Complexes Obtained from the B3LYP Functional with $6-31 \mathrm{G}(\mathrm{d}, \mathrm{p})$ and $6-31+\mathrm{G}(\mathrm{d}, \mathrm{p})$ Basis Sets: Comparison with MP2/6-31+G(d,p) Results and Experimental Data, J. Phys. Chem., 1995, 99, 10705-10707.

14 M. J. Frisch, G. W. Trucks, H. B. Schlegel, G. E. Scuseria and M. A. Robb, Gaussian 09, revision A, 02, Gaussian, Inc, Wallingford, CT, 2009.

15 H. E. Kissinger, Reaction Kinetics in Differential Thermal Analysis, Anal. Chem., 1957, 29, 1702-1706.

16 T. B. Ozawa, Kinetics in differential thermal analysis, Chemical Society of Japan, 1965, 38, 1881.

$17 \mathrm{~J}$. W. Ochterski, Thermochemistry in GAUSSIAN, Gaussian Inc, 2000.

18 M. J. Kamlet and S. J. Jacobs, Chemistry of detobation. I. Simple method for calculating detonation properties of CHON explosives, J. Chem. Phys., 1968, 48, 23.

19 M. J. Kamlet and S. J. Jacobs, Chemistry of detobation. IV. Evaluation of a simple prediction method for detonation velocities of explosives, J. Chem. Phys., 1968, 48, 3685. 